\title{
Tetra Point Wetting at the Free Surface of Liquid Ga-Bi
}

\author{
P. Huber, ${ }^{1}$ O. G. Shpyrko, ${ }^{1}$ P. S. Pershan, ${ }^{1}$ B. M. Ocko, ${ }^{2}$ E. DiMasi, ${ }^{2}$ and M. Deutsch ${ }^{3}$ \\ ${ }^{1}$ Department of Physics, Harvard University, Cambridge, Massachusetts 02138 \\ ${ }^{2}$ Department of Physics, Brookhaven National Laboratory, Upton, New York 11973 \\ ${ }^{3}$ Department of Physics, Bar-Ilan University, Ramat-Gan 52900, Israel
}

(Received 13 April 2002; published 28 June 2002)

\begin{abstract}
A continuous surface wetting transition, pinned to a solid-liquid-liquid-vapor tetra coexistence point, is studied by x-ray reflectivity in liquid Ga-Bi binary alloys. The short-range surface potential is determined from the measured temperature evolution of the wetting film. The thermal fluctuations are shown to be insufficient to induce a noticeable breakdown of mean-field behavior, expected in short-range-interacting systems due to their $d_{u}=3$ upper critical dimensionality.
\end{abstract}

DOI: $10.1103 /$ PhysRevLett.89.035502

PACS numbers: 61.25.Mv, 61.10.-i, 68.03.-g

The wetting transition, predicted independently by Cahn [1] and by Ebner and Saam [2] in 1977, has attracted much theoretical [3] and experimental [4] attention, due to its importance for fundamental physics [3] and for applications [5]. Almost all experimental and theoretical studies of the wetting transition published to date address liquids interacting through long-range van der Waals potentials, for which the upper critical dimensionality is $d_{u}<3$. Thus, for a $d=2$ surface on $d=3$ bulk mean-field (MF) behavior is expected. By contrast, wetting phenomena in liquids interacting by a short-range potential (SRW), where $d_{u}=3$, allow one, in principle, to explore the regime where the MF behavior breaks down due to fluctuations, and the renormalization-group (RG) approach becomes applicable. However, due to extremely demanding experimental requirements and the lack of a theory allowing one to predict the SRW interaction parameters, only a single experimental study addressing fluctuation effects on SRW has been published to date [6]. Even that study employs van der Waals interacting liquids, and SRW was achieved by tuning the wetting temperature, $T_{W}$, very close to the critical point, $T_{C}$. The authors' estimates of the fluctuations' magnitude predicted a RG behavior. Nevertheless, a MF behavior was found and interpreted as an "apparent failure" of RG theory to account for SRW.

We present here an x-ray study of wetting in a Ga-Bi binary alloy, which interacts by a short-range screened Coulomb potential at all concentrations. This allows achieving SRW far from $T_{C}$, thus minimizing any possible influence of the criticality on the wetting transition. Moreover, the x-ray techniques employed allowed the determination of the wetting layer's internal structure on an angström scale and demonstrated the strong compositional gradient within the wetting film, predicted by density functional calculations [2,7]. The wetting transition in this system is pinned to a bulk monotectic temperature, $T_{M}$, creating a rare case of tetra point wetting, where four phases coexist in the bulk: a Ga-rich liquid, a Bi-rich liquid, a solid $\mathrm{Bi}$, and a vapor [8,9]. We determined the temperature variation of the wetting layer's composition, thickness, and internal structure along a trajectory in the phase diagram which includes several phase boundaries and the tetra point. The study highlights the intimate relation between the wetting layer's structure and the bulk phase diagram. The $T$ dependence of the wetting film's structure yields the first quantitative determination of a MF short-range surface potential governing the wetting transition. Finally, from a RG analysis we estimate the influence of thermal fluctuations on the observed tetra point wetting.

The measured [10] bulk phase diagram of $\mathrm{Ga}-\mathrm{Bi}$ is shown in Fig. 1, with cartoons relating the surface phases to the bulk phases. For all $T$ a Gibbs-adsorbed Bi monolayer is found at the free surface [11,12]. In region I,

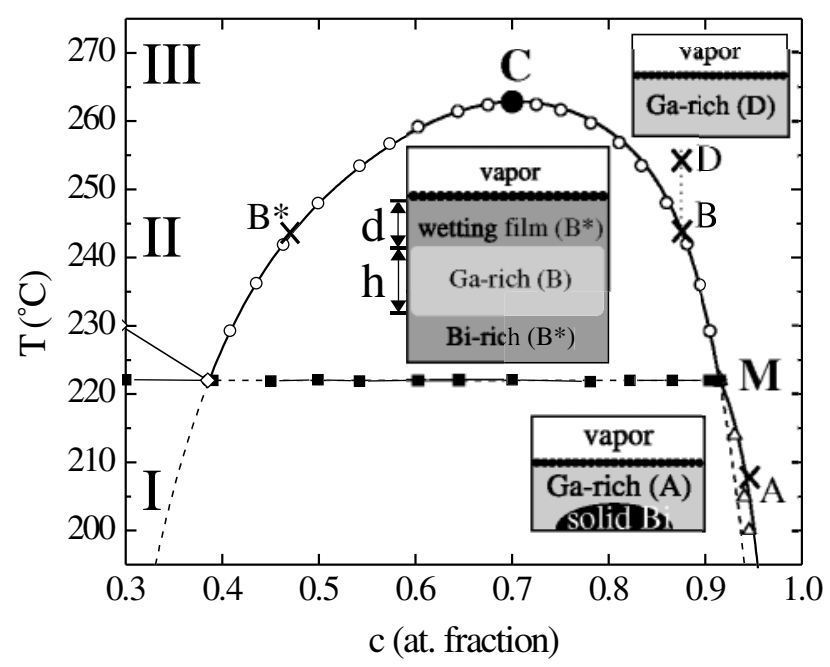

FIG. 1. The atomic fraction $(c)$, temperature $(T)$ bulk phase diagram of $\mathrm{Ga}-\mathrm{Bi}$. The symbols indicate coexistence lines of Predel's phase diagram [10]. The lines show the phase boundaries calculated from thermodynamic data [13]. The dashed lines represent the metastable extension of the $1 / 1$ coexistence line below $T_{M}$. The points are $C$, bulk critical point; $M$, monotectic point; $A, B, D$ points on the experimental path. The insets illustrate the surface and bulk phases. In region II the wetting film is $50 \AA$ and the Ga-rich fluid is $5 \mathrm{~mm}$ thick. The bold surface lines in the insets symbolize the Bi monolayer. 
$T<T_{M}$, the Bi monolayer and the solid Bi coexist with a Ga-rich liquid. In region II, $T_{M}<T<T_{C}$, the bulk separates macroscopically into immiscible Ga-rich and Bi-rich liquids, and the denser Bi-rich phase sinks to the bottom of the sample pan. However, the Bi-rich phase also wets the free surface by intruding between the Ga-rich low density phase and the $\mathrm{Bi}$ monolayer, in defiance of gravity. In the homogeneous part of the phase diagram, region III, only the Bi monolayer is found at the free surface [14]. The four phases, solid Bi, Bi-rich liquid, Ga-rich liquid, and vapor, coexist at the boundary between regions I and II, $T_{M}$, rendering it a solid-liquid-liquid-vapor tetra point.

$\mathrm{X}$-ray reflectivity measurements were carried out at beam line X22B, NSLS, at a wavelength $\lambda=1.54 \AA$. The intensity $R\left(q_{z}\right)$ reflected from the surface is measured as a function of the normal component $q_{z}$ of the momentum transfer and allows the determination of the surface-normal electron density profile $\rho(z)$ [15]. The Ga-Bi alloy was prepared in an inert-gas box using $>99.9999 \%$ pure metals. A solid Bi pellet was covered by an amount of liquid Ga required for a nominal concentration $c_{\text {nom }}=88$ at. $\% \mathrm{Ga}$. It was then transferred in air into an ultrahigh vacuum chamber. A day of bakeout yielded a pressure of $10^{-10}$ torr. The residual surface oxide on the liquid's surface was removed by sputtering with $\mathrm{Ar}^{+}$ions. Using thermocouple sensors and an active temperature control on the sample pan and its adjacent thermal shield a temperature stability and uniformity of $\pm 0.05{ }^{\circ} \mathrm{C}$ was achieved near $T_{M}$.

X-ray reflectivity $R\left(q_{z}\right)$ was measured at selected temperatures on the path $A \rightarrow M$. The standard procedure [16] for determining $\rho(z)$ from the measured $R\left(q_{z}\right)$ is to construct a physically motivated model for $\rho(z)$ and fit its Fourier transform to the measured $R\left(q_{z}\right) / R_{F}\left(q_{z}\right) . R_{F}\left(q_{z}\right)$ is the Fresnel reflectivity from an ideally flat and sharp surface having the electron density of the Ga-rich liquid. We employ a two-box model [11], where the upper box represents the Gibbs adsorbed Bi monolayer, and the lower box represents the Bi-rich wetting film. The model also includes three roughnesses for the three interfaces: vapor/ Bi monolayer, Bi monolayer/Bi-rich film, and Bi-rich film/Ga-rich subphase. The fits (lines) to the measured (points) $R / R_{F}$ are shown in Fig. 2(a), and the corresponding $\rho(z)$ profiles are shown in Fig. 2(b). At point $A\left[T_{A}=205^{\circ} \mathrm{C}\right.$, inset of Fig. 2(a)], typical of region I, $R / R_{F}$ exhibits a pronounced maximum at $q_{z}=0.8 \AA^{-1}$, indicative of a high electron density layer at the surface. The $\rho(z)$ obtained from the fit is consistent with a segregated monolayer of pure $\mathrm{Bi}$ at the free surface $[11,12]$. As the temperature is increased towards $M$, a peak in $R / R_{F}$ starts to develop at $q_{z} \approx 0.2 \AA^{-1}$, gradually shifting to lower $q_{z}$. This behavior manifests the formation of the wetting layer and its continuous growth in thickness upon approaching $T_{M}$. For the fully formed wetting layer at $M\left(T_{M}=222.0^{\circ} \mathrm{C}\right), R / R_{F}$ shows two low- $q_{z}$ peaks, characteristic of a thick film, and the fit yields a maximal
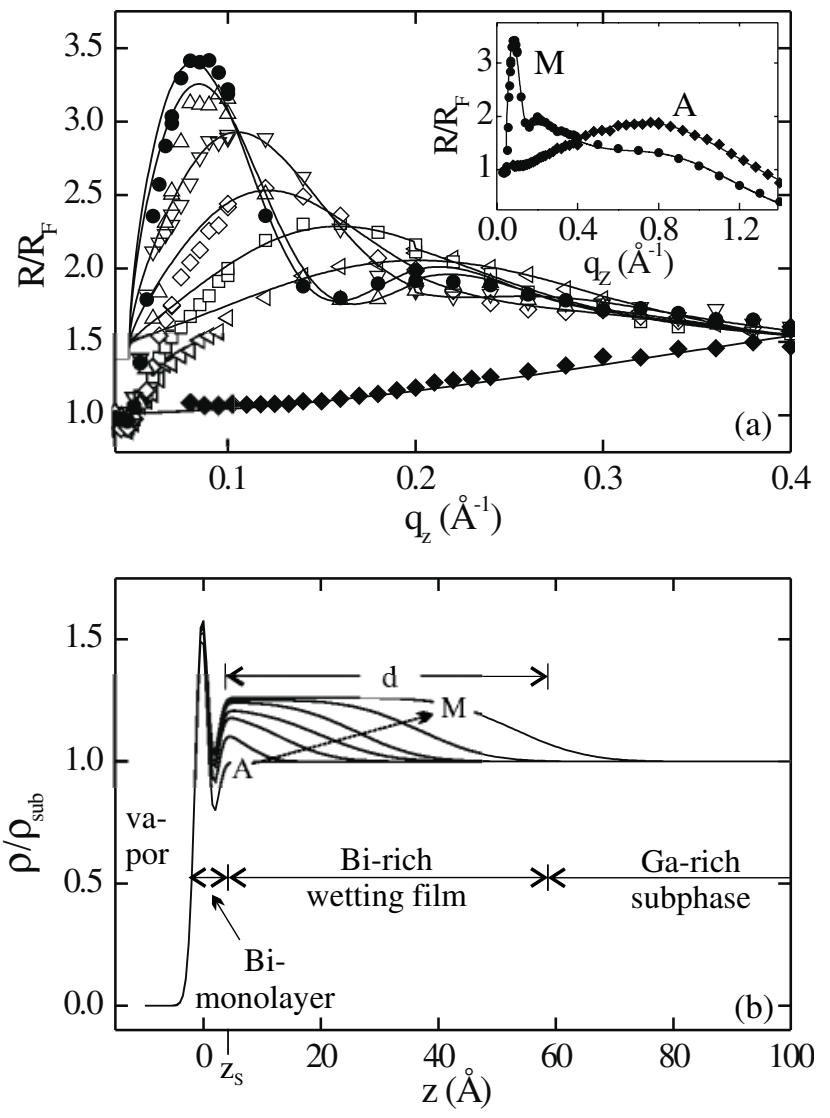

FIG. 2. (a) $R / R_{F}$ for selected temperatures $T_{A} \leq T \leq T_{M}$ : (४) $205.0^{\circ} \mathrm{C}=T_{A},(\triangleleft) 218.9^{\circ} \mathrm{C},(\square) 220.4{ }^{\circ} \mathrm{C},(\diamond) 221.0^{\circ} \mathrm{C}$, ( $\nabla) 221.5^{\circ} \mathrm{C},(\triangle) 221.8^{\circ} \mathrm{C},(\bullet) 222.0^{\circ} \mathrm{C}=T_{M}$. Solid lines: fits to a two-box model of $\rho(z)$. (b) Fit-refined electron density profiles $\rho(z)$ for aforementioned $T$ 's with $T_{A} \leq T \leq T_{M}$, $\rho_{\text {sub }}=$ electron density of Ga-rich subphase. $A$ and $M$ mark $\rho\left(T_{A}\right)$ and $\rho\left(T_{M}\right)$, respectively.

film density of $\rho / \rho_{\text {subphase }}=1.26$, as shown in Fig. 2(b). This agrees well with the value of 1.25 calculated for this ratio from the phase diagram at $T_{M}$. The fit-refined $\sim 50 \AA$ film thickness also agrees with ellipsometric measurements [17]. As can be seen in Fig. 2(b), the evolution of the density profiles along the path $A \rightarrow M$ proceeds via highly inhomogeneous wetting films that are similar to the microscopic density profiles calculated using density functional theory $[2,7]$.

The surface and bulk transitions at $T_{M}$ are driven by the excess free energy, $\Delta \mu_{m}$, of the metastable Bi-rich phase over that of the Ga-rich phase. Thus, to relate the observed continuous surface transition to the first-order bulk transition at $T_{M}$, we replot in Fig. 3(a) the $(c, T)$ phase diagram of Fig. 1 on the $\left(\Delta \mu_{m}, T\right)$ plane. For $T>T_{M}$, the $1 / 1$ coexistence line transforms into a straight line at $\left(\Delta \mu_{m}=0, T\right)$ extending from $M$ to $C$. For $T<T_{M}$ the metastable $1 / 1$ coexistence line (dashed line) extends horizontally below $T_{M}$, while the solid-Bi/Ga-rich coexistence line goes below the metastable line from $M$ to $A$. This illustrates Dietrich and Schick's observation [8] that the path leading to coexistence on heating from $A$ to $M$ is dictated 


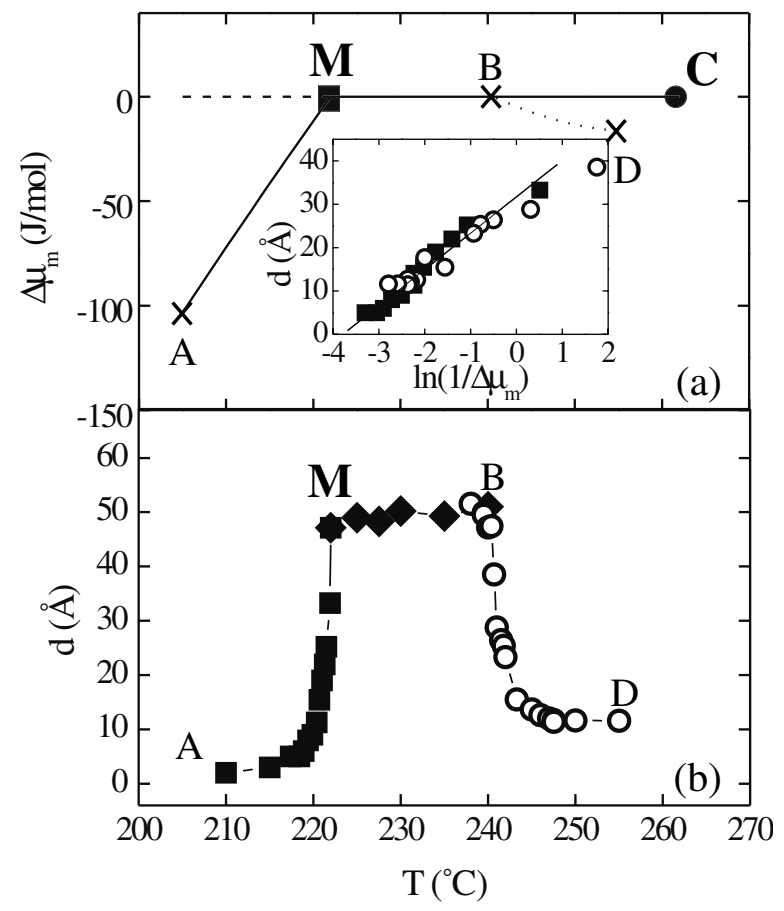

FIG. 3. (a) $\left(\Delta \mu_{m}, T\right)$ phase diagram: $(A-M)$ is the liquid-solid, and $(M-C)$ is the liquid-liquid coexistence lines. The path $B-D$ is in the single phase region, and $M$ and $C$ are the monotectic and critical points, respectively. Inset: effective wetting layer thickness $d$ on $A \rightarrow M$ (squares) and $B \rightarrow D$ (open circles). The solid line is a fit to the $A \rightarrow M d$ values. (b) The measured $d$ along the experimental path.

by the topology of the phase diagram and corresponds to a path which probes "complete wetting": $\Delta \mu_{m} \rightarrow 0$ on the path $A \rightarrow M$.

To determine the surface potential, we define an effective wetting film thickness, $d=\int_{z_{s}}^{\infty}[\rho(z)-$ $\left.\rho_{\text {Ga rich }}\right] /\left[\rho_{\text {Bi rich }}-\rho_{\text {Ga rich }}\right] d z$. Here $z_{s}$ is the top of the wetting film as marked in Fig. 2(b), and $\rho_{\text {Bi rich, }}$ $\rho_{\text {Ga rich }}$ are the electron densities of the coexisting bulk liquid phases, calculated from the phase diagram. The $d$ values calculated from the reflectivity fits along $A \rightarrow M$, $M \rightarrow B$, and $B \rightarrow D$ are plotted in Fig. 3(b). Along $A \rightarrow M$ a continuous increase in $d$ is observed, while on the on-coexistence $M \rightarrow B$ path a constant $d \approx 50 \AA$ found. This is in agreement with the predictions for SRW: a continuous $d$ growth limited by gravity effects [4]. At $B$ the $\mathrm{Ga}$ concentration reaches its nominal value, $c_{\text {nom }}=88$ at. \%, in the homogeneous liquid phase (region III). Upon further increase of $T, c$ remains unchanged. The sample leaves the $1 / 1$ coexistence line, moving farther into region III, and the wetting film vanishes continuously. Further details of the $M \rightarrow D$ path are discussed elsewhere [14].

As implied by the similarity to Ebner and Saam's [2] results, a detailed calculation of the wetting film's inhomogeneity will require either a density functional analysis or some other equivalent approach. Nevertheless, even a simple model approximating the wetting layer by a slab of thickness $d$ allows a confident determination of the surface potential. For this model, the grand canonical potential $\Omega_{S}$ at the surface is given in the MF approximation by $\Omega_{S}=N A_{0}\left[d \Delta \mu+\xi_{\mathrm{MF}} \Phi e^{-d / \xi_{\mathrm{MF}}}\right]$ with a short-range potential of decay length $\xi_{\mathrm{MF}}$ and amplitude $\Phi$. Here $\Delta \mu=\Delta \mu_{m}+\Delta \mu_{g}$ is the excess Gibbs free energy of the Bi-rich wetting phase over that of the Ga-rich phase, and $\Delta \mu_{g}=g \Delta \rho h V_{m}$ is the gravitational energy paid for having the heavier Bi-rich phase at the surface. The particle number density per unit volume is $N, A_{0}$ is an arbitrary surface area, $\Delta \rho$ is the mass density difference of the two phases, $V_{m}$ is the molar volume of the Bi-rich phase, and $h$ is the height difference between top and bottom. The equilibrium thickness, $d^{*}$, is that minimizing $\Omega_{S}:\left.\frac{\partial \Omega_{S}}{\partial d}\right|_{d^{*}}=0$ [3]. Neglecting the gravitational term for points off $1 / 1$ coexistence yields $d^{*}=\xi_{\mathrm{MF}} \ln \left(\Phi / \Delta \mu_{m}\right)$. The inset of Fig. 3(a) shows the experimental $d$ values on the path $A \rightarrow M$ (solid squares). The clear linear dependence on $\ln (1 / \Delta \mu)$ confirms the major theoretical predictions for SRW: a continuous growth and a logarithmic divergence of the wetting film's thickness [3]. A fit of $d^{*}$ (line) to the experimental $d$ (solid points) yields $\xi_{\mathrm{MF}}=6.3 \AA$ and $\Phi=43 \mathrm{~J} / \mathrm{mol}$. Both values are reasonable for such a metallic system. The value of $\Phi$ corresponds to a change in surface energy upon wetting of about $400 \mathrm{~mJ} \mathrm{~m}^{-2}$ which is consistent with surface energy measurements on $\mathrm{Ga}-\mathrm{Bi}$ alloys [18] and $\mathrm{Ga}-\mathrm{Pb}$ alloys [9]. It is interesting to compare the behavior of $d$ for the path $A \rightarrow M$ with that along the path $B \rightarrow D$, shown in the inset of Fig. 3 (open circles). The values overlap for most of the path, demonstrating that the behavior along $A \rightarrow M$ is the same as can be observed for any other path probing complete wetting, e.g., the path $B \rightarrow D$ which is solely determined by the choice of $c_{\text {nom }}$.

The analysis above exhibits very good agreement of the experimental SRW results with the universal behavior predicted by MF theory. However, it is important to note that this does not automatically mean a failure of the $\mathrm{RG}$ predictions for a nonuniversal SRW behavior in our, and other, $d_{u}=3$ systems. Both MF and RG approaches yield here a continuous, logarithmic divergence of $d^{*}$ at $T_{W}$, but with different amplitudes for $d^{*}[3,19]$. For MF, the amplitude is $\xi_{\mathrm{MF}}$ while for RG, where $d^{*} \sim \xi_{\mathrm{RG}}(1+$ $\omega / 2) \ln (1 / \Delta \mu)$, the amplitude is $\xi_{\mathrm{RG}}(1+\omega / 2)$. The "capillary parameter" $\omega=k_{B} T_{W} /\left(4 \pi \xi_{b}^{2} \gamma\right.$ ) (where $\xi_{b}$ is the bulk correlation length, and $\gamma$ is the $1 / 1$ interfacial tension) measures the magnitude of the dominant thermal fluctuations in the system, the thermally induced capillary waves at the $1 / 1$ interface of the coexisting Bi- and Ga-rich liquids. Hence, $\omega$ measures the deviation from a MF behavior, which, of course, neglects all fluctuations. A calculated [20] $\gamma=4 \mathrm{~mJ} \mathrm{~m}^{-2}$ and a $\xi_{b}=6.1 \pm 1 \AA$, estimated from the two-scale factor universality (TSFU) theory for bulk demixing in Ga-Bi [21], yield $\omega\left(T_{M}\right)=$ $0.3 \pm 0.2$. This is well within the $\omega<2$ range, where RG analysis predicts only a mild effect on complete wetting [19]. Indeed, $\xi_{\mathrm{RG}}=5.4 \AA$ is only $\sim 15 \%$ smaller 
than $\xi_{\mathrm{MF}}=6.3 \AA$ above. The two values are close, and in reasonable agreement with the value of $\xi_{b}$ expected from the TSFU [3]. Thus, a clear distinction between RG and MF behavior cannot be drawn in our case.

Finally, we compare the measured gravity-limited maximal thickness of $50 \AA M \rightarrow B$ with theory. On coexistence $\Delta \mu_{m}=0$ and thus $d_{g}^{*}=\xi_{\mathrm{RG}} \ln \left(\Phi / \Delta \mu_{g}\right)$. Using $\Phi=43 \mathrm{~J} / \mathrm{mol}$ and the known material constants in $\Delta \mu_{g}$ yields $d_{\text {grav }}^{*}=15.6 \xi_{\mathrm{RG}} \approx 85 \AA$. Since this analysis does not take into account the excess energy associated with concentration gradients across the interfaces, some overestimation of $d_{g}^{*}$ is not too surprising. However, even this rough calculation shows that the wetting film thickness is expected to be on a mesoscopic rather than on the macroscopic length scale observed for similar wetting geometries in systems governed by long-range dispersion forces [4].

We presented here the first detailed angström resolution study of a short-range wetting transition at the free surface of the binary Ga-Bi liquid alloy. The characteristic properties of SRW, a logarithmic divergence of the wetting layer's thickness upon approaching the transition, are clearly exhibited by the measurements. The observed strongly inhomogeneous internal structure of the wetting layer is similar to that obtained from density functional calculations of wetting at a hard wall $[2,7]$. The surface wetting transition is found to be pinned to the bulk monotectic point $M$ in this alloy, and the topology of the bulk phase diagram enforces a path probing complete wetting [8]. Since this path ends in $M$ where four phases coexist, the surface transition constitutes a rare tetra point wetting effect. An estimation of the capillary parameter $\omega$ indicates that fluctuations affect the observed complete SRW transition only marginally. A critical wetting transition with a similar value of $\omega$ should show more pronounced deviations from MF behavior. Here, however, this critical transition occurs on the metastable extension of the $1 / 1$ coexistence line somewhere below $T_{M}$, and may be reachable only if the alloy supercools sufficiently. Because of the mild effect of the fluctuations, the surface potential driving the transition could be determined quantitatively. This is of particular importance since in contrast with van der Waals interacting liquids, where the surface potential can be estimated from the DzyaloshinskiLifshitz-Pitaevskii theory [4,22], no equivalent theory is available for liquids with short-range interaction. We hope that this first quantitative determination of the SRW potential will stimulate the development of such theories. Available predictions, in turn, would initiate more experimental studies of fluctuation effects in SRW. In particular, they may allow a quantitative investigation of Parry's prediction of an additional renormalization of $\omega$ in SRW due to a coupling between the fluctuations at the liquid-vapor and at the $1 / 1$ interfaces [23]. This coupling may resolve the dichotomy between RG prediction and the observations in the single previous experimental study of SRW [6].

We thank Professor S. Dietrich and Professor B.I. Halperin for helpful discussions. This work is supported by U.S. DOE Grant No. DE-FG02-88-ER45379, NSF Grant No. NSF-DMR-98-72817, and the U.S.-Israel Binational Science Foundation, Jerusalem. BNL is supported by U.S. DOE Contract No. DE-AC02-98CH10886. P. H. acknowledges support from the Deutsche Forschungsgemeinschaft.

[1] J. W. Cahn, J. Chem. Phys. 66, 3667 (1977).

[2] C. Ebner and W. F. Saam, Phys. Rev. Lett. 38, 1486 (1977).

[3] P. G. de Gennes, Rev. Mod. Phys. 57, 827 (1985); S. Dietrich, in Phase Transitions and Critical Phenomena, edited by C. Domb and J.L. Lebowitz (Academic, New York, 1988), Vol. 12.

[4] B. M. Law, Prog. Surf. Sci. 66, 159 (2001); D. Bonn and D. Ross, Rep. Prog. Phys. 64, 1085 (2001).

[5] H. Gau et al., Science 283, 46 (1999); K. Kargupta et al., Phys. Rev. Lett. 86, 4536 (2001).

[6] D. Ross et al., Nature (London) 400, 737 (1999); D. Ross et al., Phys. Rev. Lett. 87, 176103 (2001).

[7] H. T. Davis, Statistical Mechanics of Phases, Interfaces and Thin Films (Wiley-VCH, New York, 1996).

[8] S. Dietrich and M. Schick, Surf. Sci. 382, 178 (1997).

[9] C. Serre et al., Surf. Sci. 415, 336 (1998).

[10] P. Predel, Z. Phys. Chem. Neue Folge 24, 206 (1960).

[11] H. Tostmann et al., Phys. Rev. Lett. 84, 4385 (2000).

[12] N. Lei et al., J. Chem. Phys. 104, 4802 (1996).

[13] The phase boundaries were calculated using data from the CalPhaD initiative (L. Kaufmann, Massachusetts Institute of Technology).

[14] P. Huber et al. (to be published).

[15] P. S. Pershan and J. Als-Nielsen, Phys. Rev. Lett. 52, 759 (1984).

[16] P. S. Pershan, Phys. Rev. E 50, 2369 (1994).

[17] D. Nattland et al., J. Phys. C 7, L457 (1995).

[18] K. B. Khokonov et al., Sov. Electrochem. 10, 865 (1974).

[19] E. Brezin et al., Phys. Rev. Lett. 50, 1387 (1983); D. M. Kroll et al., Phys. Rev. B 32, 1862 (1985).

[20] The $1 / 1 \gamma$ is calculated from the measured profile using the Cahn-Hillary theory [14].

[21] H. Kreuser and D. Woermann, J. Chem. Phys. 98, 7655 (1993).

[22] I. E. Dzyaloshinski et al., Adv. Phys. 10, 165 (1961).

[23] A. O. Parry, J. Phys. C 8, 10761 (1996). 